\title{
A water-forming NADH oxidase regulates metabolism in anaerobic fermentation
}

\author{
Xin-Chi Shi ${ }^{\dagger}$, Ya-Nan Zou ${ }^{\dagger}$, Yong Chen ${ }^{*}$, Cheng Zheng, Bing-Bing Li, Jia-Hui Xu, Xiao-Ning Shen \\ and Han-Jie Ying ${ }^{*}$
}

\begin{abstract}
Background: Water-forming NADH oxidase can oxidize cytosolic NADH to NAD ${ }^{+}$, thus relieving cytosolic NADH accumulation in Saccharomyces cerevisiae. Previous studies of the enzyme were conducted under aerobic conditions, as $\mathrm{O}_{2}$ is the recognized electron acceptor of the enzyme. In order to extend its use in industrial production and to study its effect on anaerobes, the effects of overexpression of this oxidase in S. cerevisiae BY4741 and Clostridium acetobutylicum 428 (Cac-428) under anaerobic conditions were evaluated.
\end{abstract}

Results: Glucose was exhausted in the NADH oxidase-overexpressing S. cerevisiae strain (Sce-NOX) culture after $26 \mathrm{~h}$, while $43.51 \pm 2.18 \mathrm{~g} / \mathrm{L}$ residual glucose was left in the control strain (Sce-CON) culture at this time point. After $30 \mathrm{~h}$ of fermentation, the concentration of ethanol produced by Sce-NOX reached $36.28 \pm 1.81 \mathrm{~g} / \mathrm{L}$, an increase of $56.38 \%$ as compared to Sce-CON $(23.20 \pm 1.16 \mathrm{~g} / \mathrm{L})$, while the byproduct glycerol was remarkably decreased in the culture of Sce-NOX. In the case of the C. acetobutylicum strain (Cac-NOX) overexpressing NADH oxidase, glucose consumption, cell growth rate, and the production of acetone-butanol-ethanol (ABE) all decreased, while the concentrations of acetic acid and butyric acid increased as compared to the control strain (Cac-CON). During fermentation of Cac-CON and Cac-NOX in 100-mL screw-capped bottles, the concentrations of ABE increased with increasing headspace. Additionally, several alternative electron acceptors in C. acetobutylicum fermentation were tested. Nitroblue tetrazolium and 2,6-dichloroindophenol were lethiferous to both Cac-CON and Cac-NOX. Methylene blue could relieve the effect caused by the overexpression of the NADH oxidase on the metabolic network of $C$. acetobutylicum strains, while cytochrome $c$ aggravated the effect.

Conclusions: The water-forming NADH oxidase could regulate the metabolism of both the S. cerevisiae and the C. acetobutylicum strains in anaerobic conditions. Thus, the recombinant $S$. cerevisiae strain might be useful in industrial production. Besides the recognized electron acceptor $\mathrm{O}_{2}$, methylene blue and/or the structural analogs may be the alternative elector acceptor of the NADH oxidase in anaerobic conditions.

Keywords: Alternative elector acceptor, Anaerobic fermentation, Clostridium acetobutylicum, NADH oxidase, Saccharomyces cerevisiae

\section{Background}

In Saccharomyces cerevisiae, overflow metabolism begins when the glucose uptake rate exceeds a threshold, and results in the formation of ethanol and glycerol. Glycerol

\footnotetext{
*Correspondence: chenyong1982@njtech.edu.cn; yinghanjie@njtech.edu.cn

${ }^{\dagger}$ Xin-Chi Shi and Ya-Nan Zou contributed equally to this work State Key Laboratory of Materials-Oriented Chemical Engineering, College of Biotechnology and Pharmaceutical Engineering, Nanjing Tech University, No. 30, Puzhu South Road, Nanjing 210009, People's Republic of China
}

is generated to reoxidize surplus cytosolic NADH that can accumulate during glycolysis because of the rapid consumption of glucose. Cytosolic NADH is reoxidized by two cytosolic NADH dehydrogenases, but when glycolytic NADH generation surpasses the rate at which these dehydrogenases can act, S. cerevisiae activates the glycerol synthesis pathway as another outlet for NADH consumption [1].

Recently, the water-forming NADH oxidase-encoding noxE gene from Lactococcus lactis has attracted 
substantive attention as it can relieve cytosolic NADH accumulation in $S$. cerevisiae, which does not possess this gene $[2,3]$. The use of the heterologous enzyme likely invokes an unbiased response instead of affecting a specific metabolic reaction, which will have localized network effects around the altered reaction [4]. Previous studies showed that overexpression of the water-forming $\mathrm{NADH}$ oxidase could increase the consumption of glucose and decrease the accumulation of glycerol in aerobic fermentation [1, 2]. All studies of this enzyme have been conducted under aerobic conditions, because $\mathrm{O}_{2}$ is recognized as the optimal electron acceptor of the enzyme. However, aerobic fermentation is unfit for industrial production as the air sparging will remove a large amount of ethanol. Consequently, this study aimed to investigate whether the oxidase works under anaerobic conditions.

Previous studies on Clostridium acetobutylicum and Clostridium aminovalericum, both obligatory anaerobes, showed that these two strains could grow normally under microoxic (sparged with $3 \% \mathrm{O}_{2} / 97 \% \mathrm{~N}_{2}$ mixed carrier gas) conditions $[5,6]$. Clostridium aminovalericum has a noxA gene, which was strongly upregulated when the growth conditions changed to microoxic, indicating that NoxA is involved in oxygen metabolism. In C. acetobutylicum, which has no ortholog of C. aminovalericum noxA, Northern blot analysis identified multiple $\mathrm{O}_{2}$-responsive genes that were quickly expressed or upregulated when $5 \% \mathrm{O}_{2}$ was present.

Instead of oxygen, the water-forming NADH oxidase may have alternative electron acceptors in anaerobic conditions. Although no study has focused on the alternative electron acceptor of the water-forming $\mathrm{NADH}$ oxidase to date, there are a few reports on the $\mathrm{H}_{2} \mathrm{O}_{2}$-forming NADH oxidase [7, 8]. Park et al. reported that the $\mathrm{H}_{2} \mathrm{O}_{2}$-forming $\mathrm{NADH}$ oxidase purified from the extreme thermophile Thermus thermophilus is able to catalyze electron transfer from NADH to various other electron acceptors (methylene blue, cytochrome c, $p$-nitroblue tetrazolium, 2,6-dichloroindophenol, and potassium ferricyanide).

Based on the previous findings, a S. cerevisiae strain (Sce-NOX) overexpressing a heterologous water-forming NADH oxidase was constructed. Batch culture growth of the control strain (Sce-CON) and Sce-NOX was compared. To study the role of the NADH oxidase in anaerobic bacteria, the enzyme was overexpressed in C. acetobutylicum, a strictly anaerobic gram-positive bacterium [9]. Batch culture growth of the control strain (Cac-CON) and the strain overexpressing NADH oxidase (Cac-NOX) were compared under different oxygen supply conditions. To the best of our knowledge, this is the first study to assess the role of the NADH oxidase in anaerobic condition. Our results showed that overexpression of the NADH oxidase could regulate the metabolism of both the S. cerevisiae and the C. acetobutylicum strains in anaerobic condition, which can be generalized to other strains.

\section{Methods \\ Construction of the strains}

The strains and plasmids used in this study are listed in Table 1. For the $S$. cerevisiae strain, the noxE gene from L. lactis (GenBank Accession No. AM406671) was PCRamplified with primers Sce-Nox-F, 5'-CTTGTGGG CCCAGGATCCATGAAAATCGTAGTTATCG- ${ }^{\prime}$, and Sce-Nox-R， 5'-ACAGGAATTCACCATGGATCCTTATT TGGCATTCAAAGCTG- ${ }^{\prime}$. Both primers have a BamHI site (underlined), and the homologous arms of the plasmid are indicated in italics in the primer sequences. PCR products were gel-purified and inserted into the $\mathrm{BamHI}$ site of plasmid pYX212 by using the ClonExpress ${ }^{\mathrm{TM}}$ One Step Cloning Kit (Vazyme Biotech Co., Ltd, Nanjing, China), resulting in pYX212-NOX. The plasmid was transformed into the host strain, BY4741, using G418 (400 $\mu \mathrm{g} /$ $\mathrm{mL}$ ) to select a stably transfected clone, designated SceNOX (Table 1). As a control, Sce-CON, the host strain transfected with empty plasmid was used.

For the $C$. acetobutylicum strain, the noxE gene (AM406671) was PCR-amplified with primers Cac-NoxF, 5'-CTGCAGGTCGACGGATCCATGAAAATCGTAG TTATC-3', and Cac-Nox-R, 5'-TATAGAATTCCCGG GGATCCTTATTTGGCATTCAAAGCTG-3' . Both primers have a $B a m H I$ site (underlined) and the homologous arms of the plasmid are indicated in italics in the primer sequences. Gel-purified PCR products were inserted into the BamHI site of pSY8 by using the ClonExpress ${ }^{\mathrm{TM}}$ One Step Cloning Kit, resulting in pSY8-NOX. The plasmid was transformed into C. acetobutylicum 428 (CGMCC No. 5234) using thiamphenicol $(15 \mu \mathrm{g} / \mathrm{mL})$ for clone selection. The strains containing the overexpression and empty plasmid were designated $\mathrm{Cac}-\mathrm{NOX}$ and $\mathrm{Cac}-\mathrm{CON}$, respectively (Table 1 ).

\section{Media and growth conditions}

The yeast strains (Sce-CON, Sce-NOX) were maintained on conventional yeast extract peptone dextrose (YPD) agar plates as described previously $[10,11]$.

The aerobic seed cultures for cultivation were grown at $30{ }^{\circ} \mathrm{C}$ in $500 \mathrm{~mL}$ Erlenmeyer flasks containing $100 \mathrm{~mL}$ of complex medium A (initial pH 5.2) containing $20 \mathrm{~g} / \mathrm{L}$ glucose, $10 \mathrm{~g} / \mathrm{L}$ tryptone (Oxoid), $5 \mathrm{~g} / \mathrm{L}$ yeast extract (Oxoid), and $9 \mathrm{~g} / \mathrm{L} \mathrm{NaCl} \mathrm{[12]} \mathrm{in} \mathrm{a} \mathrm{rotary} \mathrm{shaker} \mathrm{at} 200 \mathrm{rpm}$.

The anaerobic fermentations for Sce-CON and SceNOX were conducted in 100-/250-mL screw-capped bottles with two exhaust pipes, each of which had a filter membrane. The fermentation medium contained $90 \mathrm{~g} / \mathrm{L}$ glucose, $10 \mathrm{~g} / \mathrm{L}$ tryptone (Oxoid), $5 \mathrm{~g} / \mathrm{L}$ yeast extract 
Table 1 List of plasmids and strains used in this study

\begin{tabular}{lll}
\hline Plasmid/strain & Genotype & Source \\
\hline $\begin{array}{ll}\text { Plasmid } \\
\text { pYX212 }\end{array}$ & A gift from Pro. Yingjin Yuan (Tianjin University, Tianjin, China) \\
pYX212-NOX & $2 \mu$, TPI promoter, AMPR & This study \\
pSY8 & pYX212 with noxE from L. lactis & Stored in our laboratory \\
pSY8-NOX & pSY8 with noxE from L. lactis & This study \\
Strain & MATa;ura3;his3;leu2;met15 & A gift from Pro. Yingjin Yuan (Tianjin University, Tianjin, China) \\
S. cerevisiae BY4741 & BY4741/pYX212 & This study \\
$\begin{array}{l}\text { Sce-CON } \\
\text { Sce-NOX }\end{array}$ & BY4741/pYX212-NOX & This study \\
C. acetobutylicum 428 & Stored in our laboratory \\
Cac-CON & C. acetobutylicum 428/pSY8 & This study \\
Cac-NOX & C. acetobutylicum 428/pSY8-NOX & This study \\
\hline
\end{tabular}

(Oxoid), and $9 \mathrm{~g} / \mathrm{L} \mathrm{NaCl}$. The culture condition was $32{ }^{\circ} \mathrm{C}$ at $150 \mathrm{rpm}$. Nitrogen gas was used to flush the medium after inoculation and sampling to ensure anaerobic conditions.

For Cac-CON and Cac-NOX strains, the modified P2 medium for seed culture and the P2 medium for fermentation have been described previously [13]. Artificial dyes were added to the fermentation medium as indicated. In different oxygen supply fermentation conditions, the conditions were as follows: $500-/ 100-\mathrm{mL}$ Erlenmeyer flask sealed with eight-layer gauze; 500/100-mL Erlenmeyer flask sealed with eight-layer gauze and one piece of kraft paper; 250-/100-mL Erlenmeyer flask sealed with eight-layer gauze; $250-/ 100-m L$ Erlenmeyer flask sealed with eight-layer gauze and one piece of kraft paper; 100-/50-mL screw-capped bottle. All five fermentation cultures were incubated on a rotary shaker at $100 \mathrm{rpm}$, at $37^{\circ} \mathrm{C}$ and stewing in an incubator, respectively.

\section{Metabolite analyses}

The cell density of $S$. cerevisiae was measured at $600 \mathrm{~nm}$ using a BioMate ${ }^{\mathrm{TM}} 3$ spectrophotometer (Thermo Scientific, Waltham, MA, USA). Five milliliters of culture was centrifuged at $4000 \times g$ for $10 \mathrm{~min}$. The supernatants were used to determine the concentrations of glucose and metabolites.

The glucose and glycerol concentrations were measured by high-performance liquid chromatography (Agilent 1100 series; Hewlett-Packard, Palo Alto, CA, USA) with a refractive index detector, using a Benson BP-100 $\mathrm{Pb}^{++}$ column $(300 \times 7.8 \mathrm{~mm}$; Benson Polymeric Inc, Sparks, $\mathrm{NV}$, USA). Ultrapure water was used as the mobile phase at a flow rate of $0.4 \mathrm{~mL} / \mathrm{min}$ and the column temperature was set at $80^{\circ} \mathrm{C}$.
The solvents (acetone, butanol, and ethanol) and acids (butyric acid and acetic acid) were analyzed using a gas chromatography system (7890A GC-System, Agilent Technologies, Palo Alto, CA, USA) equipped with a flame ionization detector (FID) and a 30-m capillary column (Equity $1^{\mathrm{TM}} ; 30 \mathrm{~m} \times 0.32 \mathrm{~mm}, 1.0 \mu \mathrm{m}$ film thickness; Supelco Co, Bellefonate, PA, USA) [13].

\section{Enzyme activity}

Total NADH oxidation activity was assayed spectrophotometrically following the method of Vemuri et al. [1]. A unit of activity was defined as the quantity that catalyzed the oxidation of $1 \mu \mathrm{mol}$ of NADH per minute. Protein was quantified using the Bradford method using BSA as a standard.

\section{Quantification of intracellular NAD(P)H/NAD(P)}

Intracellular concentrations of $\mathrm{NAD}(\mathrm{P}) \mathrm{H}$ were determined using the enzyme cycling method of Liu et al. [13] with modifications. Generally, two $1 \mathrm{~mL}$ samples were taken and cells were collected and dissolved in $0.5 \mathrm{~mL}$ of $0.1 \mathrm{M} \mathrm{NaOH}$ (to assay $\mathrm{NAD}(\mathrm{P}) \mathrm{H}$ ) and $0.5 \mathrm{~mL}$ of $0.1 \mathrm{M}$ $\mathrm{HCl}$ (to assay $\mathrm{NAD}(\mathrm{P})$ ), respectively. The cell lysate was heated at $50{ }^{\circ} \mathrm{C}$ for $10 \mathrm{~min}$, cooled to $0{ }^{\circ} \mathrm{C}$, and centrifuged at $10,000 \times g$ for $10 \mathrm{~min}$. The supernatant was used for follow-up measurement.

A mixture of $100 \mu \mathrm{L}$ Tris- $\mathrm{HCl}(1 \mathrm{M}, \mathrm{pH} 7.8), 100 \mu \mathrm{L}$ 4.2 mM MTT, $150 \mu \mathrm{L} 16.6 \mathrm{mM}$ PES, and $100 \mu \mathrm{L}$ ethanol for the determination of $\mathrm{NAD}(\mathrm{H})$ or $100 \mu \mathrm{L} 60 \mathrm{mM}$ glucose 6-phosphate for the determination of NADP $(\mathrm{H})$ was sequentially added to a test tube and kept at $37{ }^{\circ} \mathrm{C}$ for $5 \mathrm{~min}$ in the dark. $\mathrm{ddH}_{2} \mathrm{O}$ and a moderate amount of supernatant $(75 \mu \mathrm{L}$ in total) were added to 96 -well plates. The plates were preheated at $37{ }^{\circ} \mathrm{C}$ for $5 \mathrm{~min}$ in a Multi-mode Detection Platform (SpectraMax Paradigm; 
Molecular Devices, CA, USA). Ten microliters of alcohol dehydrogenase $(1.5$ units $/ \mu \mathrm{L}$, for $\mathrm{NAD}(\mathrm{H}))$ or glucose 6-phosphate dehydrogenase (70 units/mL, for NADP $(\mathrm{H})$ ) was added to the mixture, and $46 \mu \mathrm{L}$ of the mixture was added to the 96-well plates to start the reaction. The absorbance at $570 \mathrm{~nm}$ was determined. The production for $\mathrm{NADH}$ was measured over $10 \mathrm{~min}$ at $2 \mathrm{~min}$ intervals, and the production for NADPH, NADP, and NAD was measured over $30 \mathrm{~min}$ at 5-min intervals.

\section{Quantitative reverse transcription (qRT)-PCR analysis}

RNA was isolated from cells as described previously [14]. Reverse transcription was performed using the AMV First Strand cDNA Synthesis Kit (Sangon Biotech, Shanghai, China) according to the manufacturer's instructions. Primer Express software was used for primer design. The analyzed genes and primers used in the analysis are listed in Table 2. qRT-PCR assays were performed with the SYBR Green PCR Master Mix (Applied Biosystems, Foster City, CA, USA) on a StepOnePlus Real-Time PCR System according to the manufacturer's instructions. Three technical replicates were included for each sample. Gene transcript levels were determined according to the $2^{-\Delta \Delta C t}$ method, using the ACT1 gene (for S. cerevisiae) [15] and a housekeeping gene-CA_C0279 (for C. acetobutylicum) [16] as reference genes for normalizing the gene expression levels. To verify qRT-PCR data, standard deviation values were calculated using Microsoft Excel (Microsoft Corporation, Redmond, WA, USA), and used to evaluate the repeatability and the effectively of these data.

\section{Results and discussion}

\section{Anaerobic fermentation by Sce-CON and Sce-NOX}

Batch culture growth of the control strain Sce-CON and the Sce-NOX strain overexpressing NADH oxidase in anaerobic condition was compared (Fig. 1). The glucose consumption rate and cell growth rate of SceNOX were higher than those of Sce-CON. The glucose was exhausted at $26 \mathrm{~h}$ of Sce-NOX culture, while $43.51 \pm 2.18 \mathrm{~g} / \mathrm{L}$ residual glucose remained in the SceCON culture at this time point. In addition, after $30 \mathrm{~h}$ of fermentation, the concentration of ethanol produced by Sce-NOX reached a peak value of $36.28 \pm 1.81 \mathrm{~g} / \mathrm{L}$, which was $56.38 \%$ higher than that of Sce-CON at the same time point $(23.20 \pm 1.16 \mathrm{~g} / \mathrm{L})$. The production of the byproduct glycerol by Sce-NOX was remarkably lower, which was in accordance with previous reports of increased assimilation of NADH in the cytosol by NADH oxidase, leading to reduced glycerol production $[1,3]$. The glycerol concentration of Sce-NOX remained below $1 \mathrm{~g} / \mathrm{L}$ in both seed culture and anaerobic fermentation. For Sce-CON, a large amount of glycerol was produced in both culture processes; the accumulation of glycerol
Table 2 genes and primers for quantitative real-time PCR

\begin{tabular}{|c|c|c|}
\hline Gene ID & Gene name & Primer sequences \\
\hline YFL039C & ACT1 (reference gene) & $\begin{array}{l}\text { F:TGGATTCCGGTGATGGTGTT } \\
\text { R:TGGCGTGAGGTAGAGAGAAACC }\end{array}$ \\
\hline L196579 & noxE & $\begin{array}{l}\text { F:TCAAAAATGGCGCAATCAAG } \\
\text { R:CCGCGTAAACATCTGGATCA }\end{array}$ \\
\hline YMR169C & ALD3 & $\begin{array}{l}\text { F: TGGCGGCTCAGTATTGGAA } \\
\text { R: CGCATTCTAGTGTGATATCCT } \\
\text { TAAGG }\end{array}$ \\
\hline YPL061W & ALD6 & $\begin{array}{l}\text { F: GACAAAGTCAACGGTA- } \\
\text { GAACAATCA } \\
\text { R: GGCTCTAAGGTGGTGAAGTTCATG }\end{array}$ \\
\hline YOL086C & $\mathrm{ADH} 1$ & $\begin{array}{l}\text { F: GAAGGTGCCGGTGTCGTT } \\
\text { R: ACCGATCTTCCAGCCCTTAAC }\end{array}$ \\
\hline YDL022W & GPD1 & $\begin{array}{l}\text { F: TCAATTTTTGCCCCGTATCTG } \\
\text { R: GATAGCTCTGACGTGTGAAT } \\
\text { CAACA }\end{array}$ \\
\hline YHL032C & GUT1 & $\begin{array}{l}\text { F: GCCCCAGCTCGTGAAACA } \\
\text { R: GGGCTTTCCGCTGGTTTTT }\end{array}$ \\
\hline YJR009C & $\mathrm{TDH} 2$ & $\begin{array}{l}\text { F: TCCAAGAAAGAGACCCAGCTAACT } \\
\text { R: GGAGTCAATGGCGATGTCAA }\end{array}$ \\
\hline YDR343C & HXT6 & $\begin{array}{l}\text { F: CGCTGCTATTGCAGAGCAAAC } \\
\text { R: CGAGTGGGAGGCTGAGTCA }\end{array}$ \\
\hline CA_C0279 & Housekeeping gene & $\begin{array}{l}\text { F: AGAAGTGGGAGCACCTGTAAAAA } \\
\text { R: CGGTTCAATCTTTCCTTCAACTTT }\end{array}$ \\
\hline L196579 & noxE & $\begin{array}{l}\text { F: TCAAAAATGGCGCAATCAAG } \\
\text { R: CCGCGTAAACATCTGGATCA }\end{array}$ \\
\hline CA_C1742 & Pta & $\begin{array}{l}\text { F: GAAATTCAGACCGGATCTTGCT } \\
\text { R: GCCGCTACTTCACTATCTATTGCA }\end{array}$ \\
\hline CA_C1743 & AskA & $\begin{array}{l}\text { F: CAATGGATATAAGTGCAGAA } \\
\text { GGTTCTA } \\
\text { R: CTTTGGTATCCCTTGCAATCATTA }\end{array}$ \\
\hline CA_P0163 & CtfA & $\begin{array}{l}\text { F: CAACCCAGATACTGGCAAAAAAC } \\
\text { R:TGCACGTATTCTTTCCACTA } \\
\text { GAGTTC }\end{array}$ \\
\hline CA_P0165 & Adc & $\begin{array}{l}\text { F: ACGCTATGGCGCCACTTAAT } \\
\text { R: TGCAAGAATGTGAGAGCTA } \\
\text { GAAACA }\end{array}$ \\
\hline CA_C3075 & Buk & $\begin{array}{l}\text { F: GGCGGACTCTTAAAGCCAATAGTA } \\
\text { R: GCATGCTGACCTTGAACTCCTA }\end{array}$ \\
\hline CA_C3076 & Ptb & $\begin{array}{l}\text { F: CAACACTTGATGCAGCAATGC } \\
\text { R: GCTAAAGGTCCGTCAACTACACAA }\end{array}$ \\
\hline CA_P0035 & $a d h E$ & $\begin{array}{l}\text { F: AGGGAGCAAGCGGAGATTTAT } \\
\text { R:TGCCGCATCCAAGAGTAAATG }\end{array}$ \\
\hline CA_C0028 & hydA & $\begin{array}{l}\text { F: GGAAAATGCGGAGTCTGTATGG } \\
\text { R:TGGCAACACAAGCAGCTCTAA }\end{array}$ \\
\hline
\end{tabular}

in the fermentation process was approximately five times higher than that in Sce-NOX culture.

As the glucose consumption of Sce-NOX was much faster, it can be expected that the NADH oxidase also increased the demand of NADH in anaerobic condition, since the glycolysis pathway is the main pathway to generate NADH. NADH homeostasis in response to an increase in NADH demand was achieved by the regulation of the glycolysis pathway, in accordance with a previous report of an NADPH oxidation system [17]. In our 

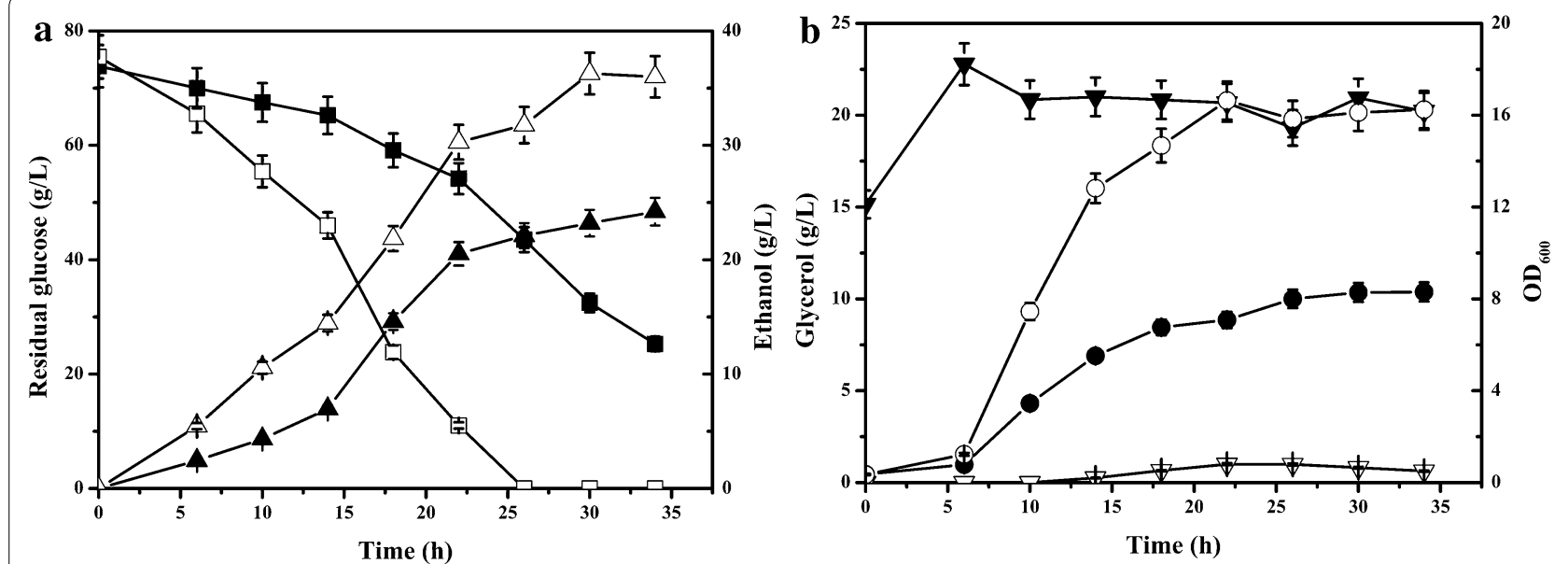

Fig. 1 Time course of fermentation by the control strain Sce-CON and the NADH oxidase-overexpressing strain Sce-NOX. a Filled triangle closed triangle, ethanol; Filled square open square, residual glucose; $\mathbf{b}$ Closed circle open circle, $\mathrm{OD}_{600} ;$ Black down-pointing triangle white down pointing triangle, glycerol. Solid symbols, Sce-CON; open symbols, Sce-NOX

batch fermentation, the concentrations of the intracellular cofactors were measured at 26,30 , and $34 \mathrm{~h}$. The intracellular $\mathrm{NADH} / \mathrm{NAD}^{+}$ratios were higher for SceNOX than for Sce-CON at all three time points, while the NADPH/NADP ${ }^{+}$ratios were similar for both strains (Fig. 2). The $\mathrm{NADH} / \mathrm{NAD}^{+}$ratios were not consistent with previously reported results. Vemuri et al. reported that for carbon-limited as well as nitrogen-limited conditions, the NADH/NAD ${ }^{+}$ratio was $20-50 \%$ lower for the NOX strain than for the control strain [1]. To confirm our results, aerobic fermentation $(500 / 100 \mathrm{~mL}$ Erlenmeyer flask sealed with eight-layer gauze; $32{ }^{\circ} \mathrm{C} ; 200 \mathrm{rpm}$ ) and microaerobic fermentation $(500 / 100 \mathrm{~mL}$ Erlenmeyer flask sealed with eight-layer gauze and one piece of kraft paper; $32{ }^{\circ} \mathrm{C} ; 150 \mathrm{rpm}$ ) were conducted. The NADH/ $\mathrm{NAD}^{+}$and NADPH/NADP ${ }^{+}$ratios in aerobic and microaerobic conditions showed the same trends as those in anaerobic conditions; the NADH/NAD ${ }^{+}$ratios of SceNOX in the three oxygen supply models were higher than those of Sce-CON (Fig. 2c-f). These inconsistencies may be due to differences between the strains used in this and the other studies. First, the parental strains were different, which may have affected the engineered phenotypes. Second, the genomic backgrounds of the NADH oxidase genes used were different. In the current study, the gene from L. lactis was used, while the gene from Streptococcus pneumoniae was used in the study by Vemuri et al. [1]. Third, the fermentation conditions were different: complete medium was used in batch fermentation in our study, while aerobic fermentations were conducted in nitrogen-limited and carbon-limited chemostats by Vemuri et al. Altogether, these differences might have led to the different results.
Because the oxidation was increased by overexpression of the NADH oxidase, one would expect increased oxidation of $\mathrm{NADH}$ and the regeneration of $\mathrm{NAD}^{+}$, leading to a decreased NADH/NAD ${ }^{+}$ratio. Thus, the increased $\mathrm{NADH} / \mathrm{NAD}^{+}$ratio in our study may seem paradoxical. A similarly paradoxical phenomenon has been reported for $S$. cerevisiae earlier; when aerobically growing cells of $S$. cerevisiae were shifted from glucose-limiting to glucose-rich conditions, the ATP level decreased by $40 \%[18,19]$. As an ample amount of phosphate is available to the cells, one would expect a new steady state to occur, accompanied by an increased ATP/ADP ratio. This phenomenon was termed the "ATP paradox." Many researchers have studied this phenomenon, using methods such as metabolic control analysis [20] and a core model consisting of a monocyclic interconvertible enzyme system [21]. Controlling the ATP concentration could be a subtle function of the relative activation of catabolic and anabolic routes. Similarly, the NADH/NAD ${ }^{+}$ratio in our study might not be determined by the single action of NADH oxidase alone, as the heterologous enzyme most probably does not affect a specific metabolic reaction. Moreover, the much faster glucose consumption of Sce-NOX might provide a larger amount of NADH, and as expected, the glycerol synthesis pathway was not activated in the presence of ample NADH. These findings indicate that the bacterial NADH oxidase has a higher affinity toward NADH than the native NADH dehydrogenases, which has been suggested by Vemuri et al. on the basis of a genome-wide transcript analysis [1]. However, the exact reason of the higher NADH/NAD ${ }^{+}$ratio observed for Sce-NOX remains unclear. 

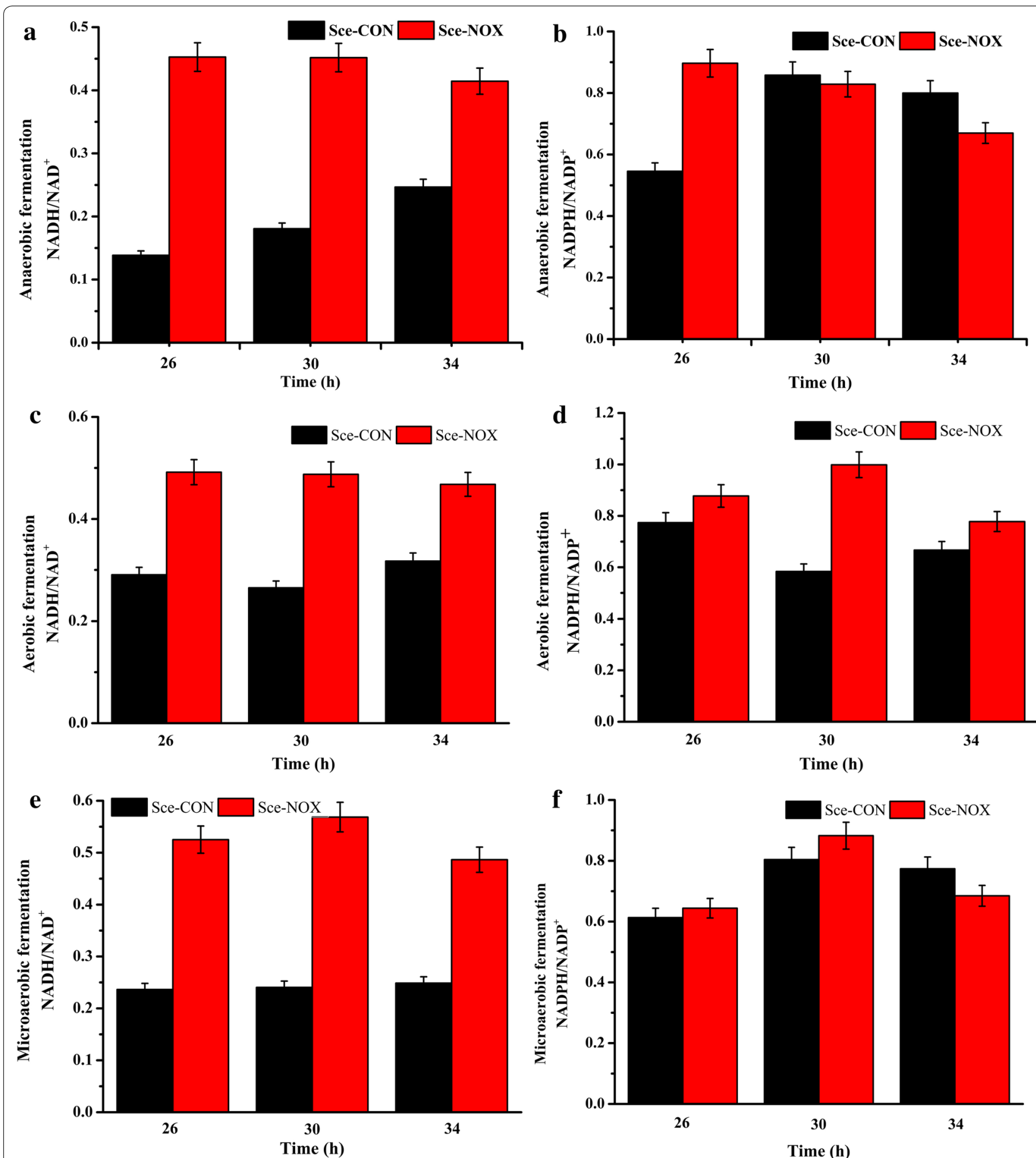

Fig. $2 \mathrm{NADH} / \mathrm{NAD}^{+}$and NADPH/NADP ${ }^{+}$ratios of Sce-CON and Sce-NOX. In the batch fermentation in different oxygen supply models, the concentrations of the intracellular cofactors of Sce-CON and Sce-NOX at 26, 30, and $34 \mathrm{~h}$ were measured. a The NADH/NAD ${ }^{+}$ratio in anaerobic fermentation; $\mathbf{b}$ The NADPH/NADP ${ }^{+}$ratio in anaerobic fermentation; $\mathbf{c}$ The NADH/NAD ${ }^{+}$ratio in aerobic fermentation; $\mathbf{d}^{\mathrm{T}} \mathrm{Th}$ NADPH/NADP ${ }^{+}$ratio in aerobic fermentation; $\mathbf{e}$ The NADH/NAD ${ }^{+}$ratio in microaerobic fermentation; $\mathbf{f}$ The NADPH/NADP ${ }^{+}$ratio in microaerobic fermentation

Next, the NADH oxidation capacity was measured. The assay for analyzing NADH oxidation is not specific for $\mathrm{NADH}$ oxidase and includes activity native to $S$. cerevisiae (e.g., NADH dehydrogenases) [1]. As shown in Fig. 3, Sce-NOX consistently exhibited greater NADH oxidation activity than Sce-CON at all three time points. 


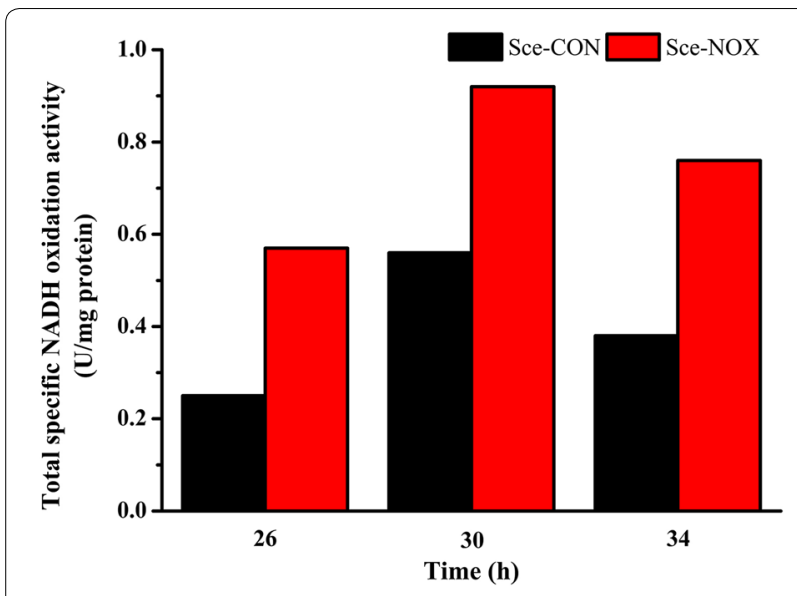

Fig. 3 Total specific NADH oxidation activity in Sce-CON and SceNOX. In the batch fermentation, the total specific NADH oxidation activity of Sce-CON and Sce-NOX at 26, 30 and $34 \mathrm{~h}$ were measured

qRT-PCR was conducted to assay the expression of $n o x E$ and genes related to ethanol and glycerol metabolism. As expected, noxE was not expressed in the control strain Sce-CON (Fig. 4a). On the other hand, it was effectively expressed in the recombinant strain Sce-NOX. The expression of GUT1, involved in the glycerol assimilation pathway, was upregulated, whereas the expression of GPD1 gene, involved in the glycerol synthesis pathway, did not substantially change. The expression of $A L D 6$ was upregulated, suggesting that the conversion of acetaldehyde to acetate was stimulated, consistent with a previous report [1]. The detailed results and the calculative process were shown in the Additional file 1.

\section{Anaerobic fermentation by Cac-CON and Cac-NOX}

Batch culture growth of the control strain $\mathrm{Cac}-\mathrm{CON}$ and of the Cac-NOX strain overexpressing NADH oxidase in anaerobic condition was compared. After $72 \mathrm{~h}$, the bubbles disappeared and the fermentation was completed. As shown in Table 3, when compared with Cac-CON, the metabolism of Cac-NOX changed obviously. The consumption of glucose and the concentrations of acetone, butanol, and ethanol (ABE) were all lower in Cac-NOX culture. For $\mathrm{Cac}-\mathrm{CON}$, the concentrations of $\mathrm{ABE}$ were 3.04, 10.43, and $6.24 \mathrm{~g} / \mathrm{L}$, respectively. For Cac-NOX, the concentrations of $\mathrm{ABE}$ were $2.17,7.24$, and $1.32 \mathrm{~g} / \mathrm{L}$, respectively, showing reductions of 28.62, 30.58, and $78.85 \%$ as compared to Cac-CON. In contrast, the concentrations of acetic acid $(1.17 \pm 0.08$ vs. $0.32 \pm 0.02 \mathrm{~g} / \mathrm{L})$ and butyric acid $(0.56 \pm 0.01$ vs. $0.11 \pm 0.01 \mathrm{~g} / \mathrm{L})$ were more than five times higher for Cac-NOX. Different from the results obtained in S. cerevisiae, the intracellular NADH/NAD ${ }^{+}$ratio of Cac-CON was $0.22 \pm 0.02$,
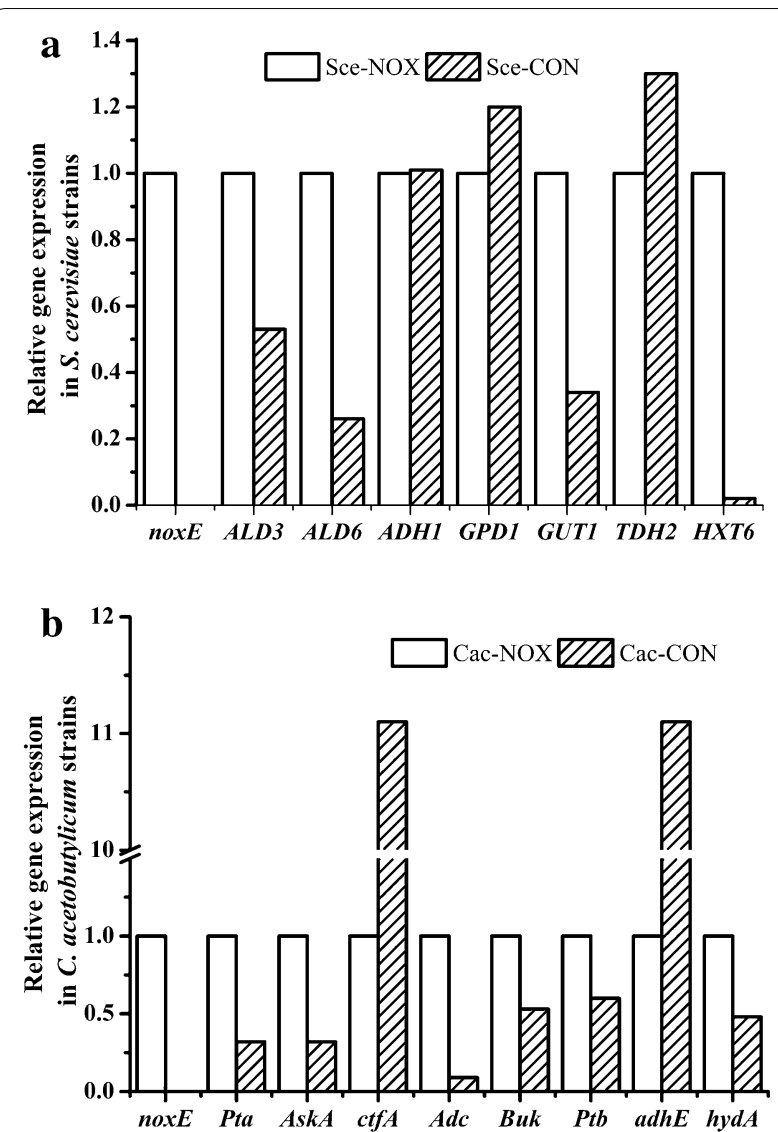

Fig. 4 qRT-PCR analysis of S. cerevisiae and C. acetobutylicum strains. Cycle threshold (Ct) values from three technical replicates were averaged. Fold differences were calculated using the reference gene ACT1 for S. cerevisiae strains the housekeeping gene CA_C0279 for C. acetobutylicum strains. a Results of qRT-PCR for genes related to ethanol and glycerol metabolism in S. cerevisiae. noxE NADH oxidase, ALD3 aldehyde dehydrogenase, ALD6 aldehyde dehydrogenase, ADH1 alcohol dehydrogenase, GPD1 glycerol-3-phosphate dehydrogenase, GUT1 glycerol kinase, TDH2 glyceraldehyde-3-phosphate dehydrogenase, HXT6 hexose transporter. b Results of qRT-PCR for genes related in ABE and acid metabolism in C. acetobutylicum. noxE NADH oxidase, Pta phosphotransacetylase, AskA acetate kinase, ctfA butyrate-acetoacetate CoA-transferase, Adc acetoacetate decarboxylase, Buk butyrate kinase, Ptb phosphate butyryltransferase, adhE bifunctional acetaldehyde-CoA/alcohol dehydrogenase, hydA hydrogen dehydrogenase

while it was $0.15 \pm 0.02$ for Cac-NOX, i.e., a $31.82 \%$ decrease. The deficiency of $\mathrm{NAD}(\mathrm{P}) \mathrm{H}$ hampered the conversion of acid into alcohol. A previous study reported that the addition of methyl viologen, which could shift the metabolism of $C$. acetobutylicum away from hydrogen production toward alcohol formation, could increase the intracellular $\mathrm{NADH} / \mathrm{NAD}^{+}$ratio [22]. However, when $\mathrm{H}_{2} \mathrm{O}_{2}$ was added into the medium, the intracellular NADH/NAD ${ }^{+}$ratio was decreased, and the expression of genes encoding $\mathrm{NADH}$-consuming enzymes in 
Table 3 Comparison of batch fermentation of Cac-CON and Cac-NOX

\begin{tabular}{llllllll}
\hline & Residual glucose (g/L) & Acetone (g/L) & Ethanol (g/L) & Butanol (g/L) & Acetoin (g/L) & Acetic acid (g/L) & Butyric acid (g/L) \\
\hline Cac-CON & $2.45 \pm 0.12$ & $3.04 \pm 0.15$ & $6.24 \pm 0.31$ & $10.43 \pm 0.52$ & $2.20 \pm 0.11$ & $0.32 \pm 0.02$ & $0.11 \pm 0.01$ \\
Cac-NOX & $26.82 \pm 1.34$ & $2.17 \pm 0.11$ & $1.32 \pm 0.07$ & $7.24 \pm 0.36$ & $0.59 \pm 0.03$ & $1.71 \pm 0.08$ & $0.56 \pm 0.01$ \\
\hline
\end{tabular}

Each value is an average of three parallel replicates

the central metabolism was repressed, increasing the availability of reducing power needed for reduction of $\mathrm{H}_{2} \mathrm{O}_{2}$ [22]. The addition of $\mathrm{H}_{2} \mathrm{O}_{2}$, similar to our operation, increased the loss of reducing power, which in turn decreased the intracellular $\mathrm{NADH} / \mathrm{NAD}^{+}$ratio and the production of alcohol. The NADH oxidation capacity in the $C$. acetobutylicum strains was measured at the end of fermentation. In Cac-CON, the NADH oxidation activity was below the detection limit, while an activity of $0.57 \mathrm{U} / \mathrm{mg}$ protein was noted for Cac-NOX. In our study, although the water-forming $\mathrm{NADH}$ oxidase did not improve the production of butanol as reported by Kawasaki et al. [5], it certainly did regulate the metabolism of C. acetobutylicum.

qRT-PCR performed to assay the expression of noxE and genes related to $\mathrm{ABE}$ and acid metabolism revealed that $n o x E$ was not expressed in Cac-CON, while it was expressed in Cac-NOX (Fig. 4b). In accordance with the results of batch fermentation, the expression of $c t f A$ and $a d h E$, which are involved in the ABE synthetic pathway, was significantly downregulated. The expression of Pta and $A s k A$, involved in acetic acid synthesis, was significantly upregulated. Finally, hydA expression was upregulated, indicating the increased loss of reducing power. The detailed results and the calculative process were shown in the Additional file 1.

Thus, overexpression of the water-forming NADH oxidase in the two different microorganisms, S. cerevisiae and $C$. acetobutylicum, could regulate the metabolism of both strains in anaerobic fermentation, confirming that the NADH oxidase has an alternative electron acceptor in anaerobic conditions, although the optimal and accepted electron acceptor of this enzyme is $\mathrm{O}_{2}$.

Differential response of S. cerevisiae and C. acetobutylicum to overexpression of the water-forming NADH oxidase

In S. cerevisiae, rapid consumption of glucose can lead to the accumulation of NADH; thus, lowering NADH accumulation by elevating either the rate of respiration or the direct oxidation of NADH is a logical approach to reduce overflow metabolism in S. cerevisiae (Fig. 5a). Glycerol is generated to reoxidize surplus cytosolic NADH that is formed in glycolysis [1]. Because of the outflow of intracellular acetaldehyde, glycerol branch shunting acts to reduce the efficiency of ATP regeneration. Shifting the end product of glucose metabolism from ethanol to glycerol abates ATP regeneration and increases ATP consumption. Therefore, the higher the glycerol accumulation, the lower the ATP regeneration efficiency is [23]. In this study, overexpression of the NADH oxidase elevated the rate of NADH oxidation in anaerobic condition. The elevated $\mathrm{NAD}^{+}$regeneration increased the flux to glycolysis, which finally increased the production of ethanol.

In $C$. acetobutylicum, the decarboxylation and dehydrogenation of pyruvate are catalyzed by ferredoxin oxidoreductase. In addition to the generation of acetyl-CoA, this process involves the reduction of ferredoxin ( $\mathrm{Fd}$ ). The reduced ferredoxin $\mathrm{FdH}_{2}$ can transfer an electron to $\mathrm{NAD}(\mathrm{P})^{+}$to generate $\mathrm{NAD}(\mathrm{P}) \mathrm{H}$, which can be further used in the synthesis of ethanol and butanol. However, in the process of butanol fermentation, a large fraction of $\mathrm{FdH}_{2}$-derived hydrogen was not used to generate intracellular NAD(P)H but escaped in the form of hydrogen by the action of hydrogenase. Hydrogen escape led to a shortage of reducing power in the fermentation. It is the root cause of substantial accumulation of by-products, such as acetone and acetic acid, and the low yield of butanol [13]. Former studies have aimed at reducing the loss of reducing power to improve the butanol production capacity [24-26]; however, in our study, increasing the oxidation of $\mathrm{NADH}$, which is equivalent to the decline of reducing power, most likely caused the accumulation of by-products and the lower production of the main products $\mathrm{ABE}$. The imbalance of redox state decreased the glucose consumption and cell growth. As is well known, the fermentation of $C$. acetobutylicum is composed of two stages: production of acid during the exponential growth phase and production of alcohol during the stationary phase [8]. The low cell growth rate and the low intracellular concentration of $\mathrm{NAD}(\mathrm{P}) \mathrm{H}$ hampered transition to the alcohol production phase as the production of ethanol and butanol requires $\mathrm{NAD}(\mathrm{P}) \mathrm{H}$ (Fig. 5b).

\section{Survivability of Cac-NOX in different oxygen supply models}

Multiple reports have mentioned that when exposed to oxygen, some anaerobic bacteria overexpress NADH oxidase to enhance their survival $[5,6,27]$. To investigate 


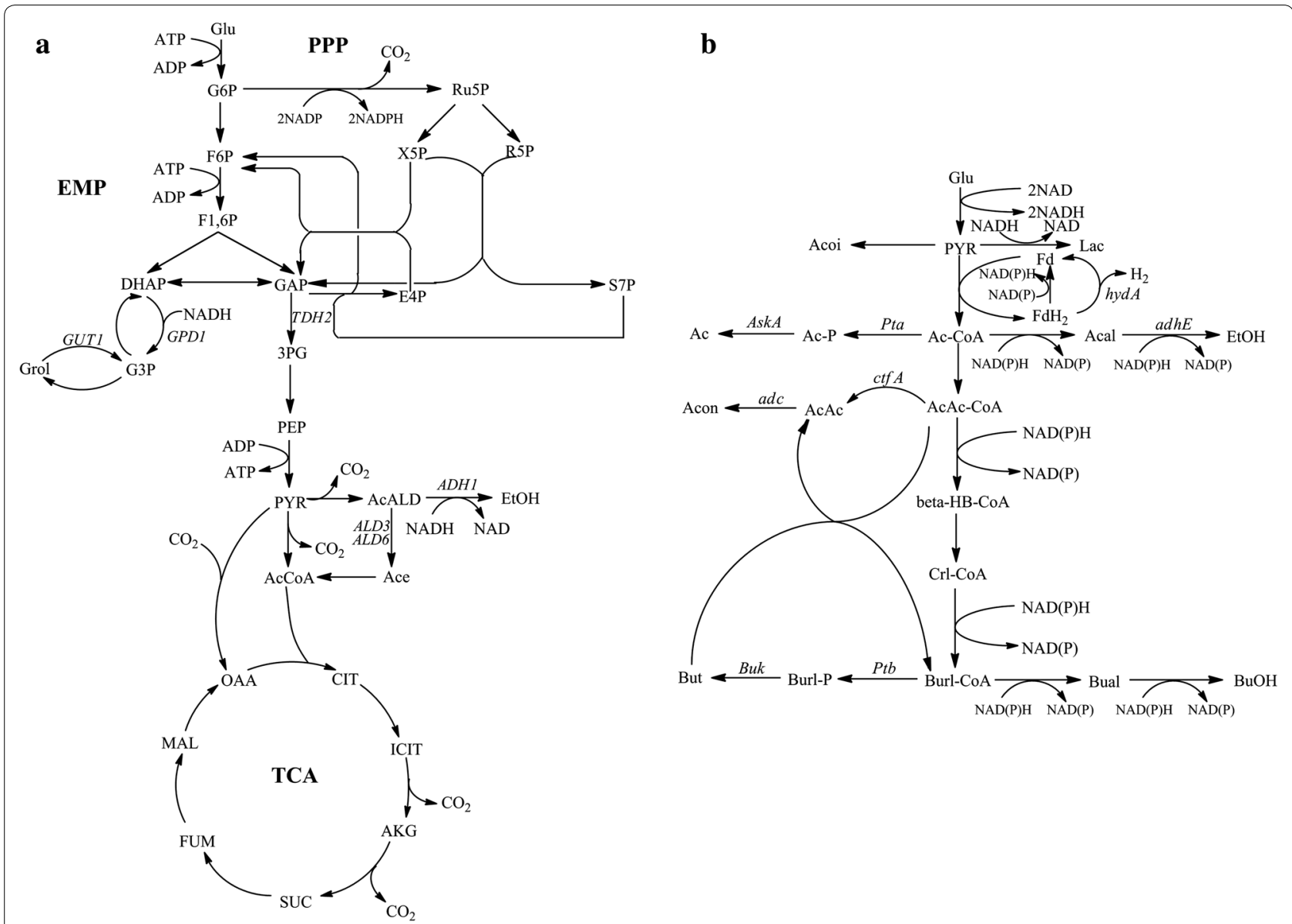

Fig. 5 Metabolic networks of S. cerevisiae and C. acetobutylicum. a The metabolic network of S. cerevisiae; b The metabolic network of C. acetobutylicum

whether the water-forming NADH oxidase might act as an oxygen scavenger in C. acetobutylicum, we designed a series of experiments in different oxygen supply conditions using the same seed culture. The results showed that, regardless of shaking, both Cac-CON and Cac-NOX showed very poor cell growth in the bottles sealed with eight layers of gauze (and one piece of kraft paper). In these conditions, the cells could not form a membrane as under the normal conditions in $100-/ 50-\mathrm{mL}$ screwcapped bottles. In addition, the consumption of glucose was quite slow, resulting in high concentrations of residual sugar (data not shown).

To determine the maximum level of oxygen supply that the $C$. acetobutylicum strains can tolerate, the effect of headspace on the growth of Cac-CON and Cac-NOX and production of solvent was studied (no nitrogen was sparged). The headspace volume fractions tested were 0, 25, 50 and $75 \%$ [11]. As shown in Table 4, different headspace had little effect on Cac-CON. ABE, acetic acid, and butyric acid were produced at the same level in all four conditions. The production of $\mathrm{ABE}$ by Cac-NOX did not exceed that by Cac-CON in any of the headspace volumes tested, while the concentrations of acetic acid and butyric acid were higher. The highest concentration of butanol $(8.79 \pm 0.44 \mathrm{~g} / \mathrm{L})$ was obtained in the case of $75 \%$ headspace. With decreasing headspace, the production of $\mathrm{ABE}$ declined while the production of acetic acid and butyric acid did not change obviously. We hypothesized above that the overexpression of the water-forming NADH oxidase in C. acetobutylicum would exacerbate the deficiency of reducing power, which would subsequently affect the production of $\mathrm{ABE}$. However, the headspace experiments showed that upon overexpression of the NADH oxidase, higher concentrations of $\mathrm{ABE}$ could be obtained with larger headsspace in the screw-capped bottle. This finding was in accordance with the results reported by Al-Shorgani et al. [11]. 
Table 4 The influence of headspace to the control strain Cac-CON and the overexpressing NADH oxidase strain Cac-NOX

\begin{tabular}{|c|c|c|c|c|c|c|c|c|}
\hline \multirow[t]{3}{*}{ Products (g/L) } & \multicolumn{8}{|l|}{ Headspace } \\
\hline & \multicolumn{4}{|l|}{ Cac-CON } & \multicolumn{4}{|l|}{ Cac-NOX } \\
\hline & $75 \%$ & $50 \%$ & $25 \%$ & $0 \%$ & $75 \%$ & $50 \%$ & $25 \%$ & $0 \%$ \\
\hline Acetone & $5.12 \pm 0.26$ & $4.47 \pm 0.22$ & $5.08 \pm 0.25$ & $4.56 \pm 0.23$ & $4.90 \pm 0.24$ & $4.18 \pm 0.21$ & $2.99 \pm 0.15$ & $1.31 \pm 0.07$ \\
\hline Ethanol & $2.07 \pm 0.10$ & $1.98 \pm 0.10$ & $2.11 \pm 0.11$ & $1.83 \pm 0.09$ & $0.77 \pm 0.04$ & $0.95 \pm 0.05$ & $0.48 \pm 0.02$ & $0.24 \pm 0.01$ \\
\hline Butanol & $12.15 \pm 0.61$ & $11.96 \pm 0.60$ & $12.17 \pm 0.61$ & $11.78 \pm 0.59$ & $8.79 \pm 0.44$ & $8.37 \pm 0.42$ & $5.84 \pm 0.29$ & $3.29 \pm 0.16$ \\
\hline Acetic acid & $0.84 \pm 0.04$ & $0.45 \pm 0.02$ & $0.52 \pm 0.03$ & $1.78 \pm 0.09$ & $1.56 \pm 0.08$ & $3.37 \pm 0.17$ & $1.91 \pm 0.10$ & $2.50 \pm 0.12$ \\
\hline Butyric acid & $0.64 \pm 0.03$ & $0.28 \pm 0.01$ & $0.24 \pm 0.01$ & $0.32 \pm 0.02$ & $1.47 \pm 0.07$ & $0.32 \pm 0.02$ & $0.74 \pm 0.04$ & $0.45 \pm 0.02$ \\
\hline
\end{tabular}

Each value is an average of three parallel replicates

\section{Alternative electron acceptors of the water-forming NADH oxidase in anaerobic condition}

Our results indicated that the water-forming NADH oxidase has alternative electron acceptors in anaerobic condition. Currently, only limited reports focus on alternative electron acceptors. The $\mathrm{H}_{2} \mathrm{O}_{2}$-forming $\mathrm{NADH}$ oxidase has alternative electron acceptors, such as methylene blue, cytochrome c, $p$-nitroblue tetrazolium, 2,6-dichloroindophenol, and potassium ferricyanide in anaerobic conditions $[7,8]$. Some of these were used in the $C$. acetobutylicum fermentation to assess whether they were the alternative electron acceptors in anaerobic conditions, as the difference between Cac$\mathrm{CON}$ and Cac-NOX was more obvious than that between Sce-CON and Sce-NOX. Methylene blue, cytochrome c, p-nitroblue tetrazolium, and 2,6-dichloroindophenol were added into the fermentation medium through filter membranes at a concentration of $0.1 \mathrm{~g} / \mathrm{L}$. In the medium with $p$-nitroblue tetrazolium or 2,6-dichloroindophenol, both the Cac-CON and Cac-NOX strains could not grow at all, and all the products were nearly undetectable (data not shown). However, both Cac-CON and Cac-NOX could grow in the presence of methylene blue or cytochrome c. Methylene blue and cytochrome c had little influence on the metabolism of Cac-CON. The production of ethanol, butanol, and acetic acid remained at the same level. Cytochrome $\mathrm{c}$ led to a slight increase in the concentration of acetone $(2.16 \pm 0.11 \mathrm{vs}$. $3.20 \pm 0.16 \mathrm{~g} / \mathrm{L}$ ) and a small decrease in that of butyric acid (3.30 \pm 0.17 vs. $1.52 \pm 0.08$ g/L) (Fig. 6). In contrast, the production of metabolites in Cac-NOX culture changed a lot in the presence of the potential electron acceptors methylene blue or cytochrome c. In the case of methylene blue, the concentrations of $\mathrm{ABE}$ were all increased while those of acetic acid and butyric acid were decreased. The concentration of butanol produced by Cac-NOX increased from $3.10 \pm 0.15$ to $4.23 \pm 0.21 \mathrm{~g} / \mathrm{L}$, and the concentration of butyric acid decreased from $8.90 \pm 0.45$ to $6.08 \pm 0.30 \mathrm{~g} / \mathrm{L}$. In the case of cytochrome

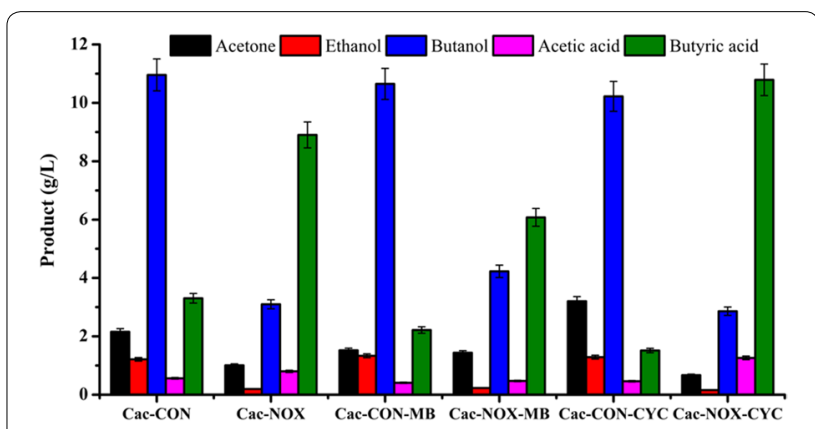

Fig. 6 Influence of the potential electron acceptors on the metabolism of $C$. acetobutylicum. The potential electron acceptors methylene blue and cytochrome c were added into the medium through filter membranes before fermentation of Cac-CON and Cac-NOX

c, the production of $\mathrm{ABE}$ was slightly reduced, while that of acetic acid and butyric acid increased. The concentration of butyric acid increased from $8.90 \pm 0.45$ to $10.79 \pm 0.54 \mathrm{~g} / \mathrm{L}$. These results showed that methylene blue could relieve the effects on the metabolic network of $C$. acetobutylicum strains, decline in reducing power, accumulation of by-products, and the lower production of the main products $\mathrm{ABE}$, caused by the overexpression of the water-forming NADH oxidase, while cytochrome $c$ aggravated the effects, possibly by exacerbating the imbalance of cofactors or the deficiency of reducing power. Methylene blue and/or the structural analogs could be the alternative elector acceptor of the waterforming NADH oxidase in anaerobic conditions.

\section{Conclusions}

This study highlighted the role of overexpression of the water-forming NADH oxidase in S. cerevisiae and C. acetobutylicum in anaerobic conditions. In contrast to previous studies, which focused on the aerobic condition, the metabolism of the two different microorganisms could be regulated by this NADH oxidase in anaerobic fermentation, showing the potential usability of the recombinant $S$. 
cerevisiae strain in large-scale ethanol production. Larger headspace was better for the growth and $\mathrm{ABE}$ production of strain Cac-NOX in the screw-capped bottle. In addition, methylene blue and/or the structural analogs could be the alternative elector acceptor of the water-forming $\mathrm{NADH}$ oxidase in anaerobic conditions. However, the detailed mechanism underlying the higher NADH/NAD ${ }^{+}$ ratio in Sce-NOX than in Sce-CON could not be determined in this study and requires further research.

\section{Additional file}

Additional file 1. Results of qRT-PCR of S. cerevisiae and C.

acetobutylicum.

\begin{abstract}
Abbreviations
Sce-CON: Saccharomyces cerevisiae BY4741 containing the empty plasmid pYX212; Sce-NOX: S. cerevisiae BY4741 overexpressing the water-forming NADH oxidase encoded by noxE; Cac-428: C. acetobutylicum 428; Cac-CON: C. acetobutylicum 428 containing the empty plasmid pSY8; Cac-NOX: C. acetobutylicum 428 overexpressing the water-forming NADH oxidase encoded by noxE; $\mathrm{ABE}$ : acetone-butanol-ethanol.
\end{abstract}

\section{Authors' contributions}

XCS participated in the design of the study, constructed the plasmids and strains, participated in the fermentation experiments, drafted the manuscript and revised the manuscript. YNZ participated in the fermentation experiments. YC participated in the design of the study. CZ helped analyze the data and revise the manuscript. BBL helped construct the plasmids and strains. JHX helped carry out the fermentation experiments. XNS helped carry out the fermentation experiments. HJY conceived of the study, and participated in its design. All authors read and approved the final manuscript.

\section{Authors' information}

Xinchi Shi received her BE in biological engineering from Nanjing Tech University, China, in 2012, and joined Prof. Ying's group pursuing her doctoral studies at the same year in biochemical engineering under the supervision of associate professor Chen and Prof. Ying. Her research interests are in the field of the impact of cofactor perturbation on S. cerevisiae.

Yanan Zou studied biological engineering in Huanyin Institute of Technology (China), where she got her Bachelor's degree in 2014. She is now pursuing her master studies at Nanjing Tech University under the supervision of associate professor Chen and Prof. Ying. Her research interests are in the field of the regulatory mechanism of the overexpressed NADH oxidase in S. cerevisiae and C. acetobutylicum.

Yong Chen studied biological engineering in Nanjing Tech University, and received his Ph.D. degree under the supervision of Prof. Ying in 2008. He then works with Prof. Ying as a teacher. His major researches include biocatalysis and enzyme engineering.

Cheng Zheng was born in Nanjing, China. He received his BSc in Nanjing Tech University. In 2013, he joined Prof. Ying's group to pursue master studies. He is now focusing on field of yeast biofilm under the supervision of associate professor Yong Chen.

Bingbing Li studied biological engineering in Henan University of Science and Technology (China), where he got his Bachelor's degree in 2010. He is now pursuing his doctoral studies at Nanjing Tech University under the supervision of Prof. Ying. His research interests are in the field of the high yield of RNA in Candida tropicalis.

Jiahui Xu was born in Jiangsu, China, in 1992. She studied pharmaceutical engineering in Nanjing Tech University (China), where she got her Bachelor's degree in 2014, and joined Prof. Ying's group pursuing her master studies at the same year. Her research interests are in the field of the formations and functions of bacterial biofilm in C. acetobutylicum.

Xiaoning Shen studied pharmaceutical engineering in Huanyin Institute of Technology (China), where she got her Bachelor's degree in 2014. She is now pursuing her master studies at Nanjing Tech University under the supervision of Prof. Ying. Her research interests are in the field of the coproduction of acetion and $\mathrm{ABE}$ by $\mathrm{C}$. acetobutylicum.

Hanjie Ying studied biotechnology in Nanjing Tech University, and received his Ph.D. degree under the supervision of Prof. Pingkai Ouyang. His major researches include bioseparation technology and enzyme engineering. He had successfully developed enzymatic methods for 1,6-FDP and 5'-nucleotide producing in industry.

\section{Acknowledgements}

We thank Yingjin Yuan for providing plasmid pYX212 and S. cerevisiae BY4741. This work was supported by the National High-Tech Research and Development Program of China (863) (2012AA021203), the National Basic Research Program of China (973) (2013CB733602), the Major Research Plan of the National Natural Science Foundation of China (21390204), the National Technology Support Program (2012BAI44G01), the National Natural Science Foundation of China, General Program (2137611), the Program for Changjiang Scholars and Innovative Research Team in University (IRT_14R28), and the Priority Academic Program Development of Jiangsu Higher Education Institutions (PAPD).

\section{Availability of supporting data}

The authors promise the availability of supporting data.

\section{Competing interests}

The authors declare that they have no competing interests.

\section{Consent for publication}

The authors have consented for publication.

\section{Ethical approval and consent to participate}

Not applicable.

Received: 8 January 2016 Accepted: 26 April 2016

Published online: 11 May 2016

\section{References}

1. Vemuri GN, Eiteman MA, McEwen JE, Olsson L, Nielsen J. Increasing $\mathrm{NADH}$ oxidation reduces overflow metabolism in Saccharomyces cerevisiae. P Natl Acad Sci USA. 2007;104(7):2402-7.

2. Heux S, Cachon R, Dequin S. Cofactor engineering in Saccharomyces cerevisiae: expression of a $\mathrm{H}_{2} \mathrm{O}$-forming $\mathrm{NADH}$ oxidase and impact on redox metabolism. Metab Eng. 2006;8(4):303-14.

3. Hou J, Lages NF, Oldiges M, Vemuri GN. Metabolic impact of redox cofactor perturbations in Saccharomyces cerevisiae. Metab Eng. 2009;11:253-61.

4. Holm AK, Blank LM, Oldiges M, Schmid A, Solem C, Jensen PR, Vemuri GN. Metabolic and transcriptional response to cofactor perturbations in Escherichia coli. J Bio Chem. 2010;285(23):17498-506.

5. Kawasaki S, Ishikura J, Chiba D, Nishino T, Niimura Y. Purification and characterization of an $\mathrm{H}_{2} \mathrm{O}$-forming $\mathrm{NADH}$ oxidase from Clostridium aminovalericum: existence of an oxygen-detoxifying enzyme in an obligate anaerobic bacteria. Arch Microbiol. 2004;181(4):324-30.

6. Kawasaki S, Watamura Y, Ono M, Watanabe T, Takeda K, Niimura Y. Adaptive responses to oxygen stress in obligatory anaerobes Clostridium acetobutylicum and Clostridium aminovalericum. Appl Environ Microb. 2005;71(12):8442-50.

7. Park HJ, Reiser CO, Kondruweit S, Erdmann H, Schmid RD, Sprinzl M. Purification and characterization of a NADH oxidase from the thermophile Thermus thermophilus HB8. Eur J Biochem. 1992;205:881-5.

8. Herles C, Braune A, Blaut M. Purification and characterization of an NADH oxidase from Eubacterium ramulus. Arch Microbiol. 2002;178(1):71-4.

9. Wietzke $M, B a h l H$. The redox-sensing protein Rex, a transcriptional regulator of solventogenesis in Clostridium acetobutylicum. Appl Microbiol biot. 2012;96(3):749-61. 
10. Ito H, Fukuda Y, Murata K, Kimura A. Transformation of intact yeast cells treated with alkali cations. J Bacteriol. 1983;153(1):163-8.

11. Al-Shorgani NKN, Kalil MS, Yusoff WMW, Hamid AA. Biobutanol production by a new aerotolerant strain of Clostridium acetobutylicum YM1 under aerobic conditions. Fuel. 2015;158:855-63.

12. Barber AR, Vriesekoop F, Pamment NB. Effects of acetaldehyde on Saccharomyces cerevisiae exposed to a range of chemical and environmental stresses. Enzyme Microb Tech. 2002;30:240-50.

13. Liu D, Chen Y, Li A, Ding F, Zhou T, He Y, Li B, Niu H, Lin X, Xie J, et al. Enhanced butanol production by modulation of electron flow in Clostridium acetobutylicum B3 immobilized by surface adsorption. Bioresource Technol. 2013;129:321-8.

14. Cao YY, Cao YB, Xu Z, Ying K, Li Y, Xie Y, Zhu ZY, Chen WS, Jiang YY. cDNA microarray analysis of differential gene expression in Candida albicans biofilm exposed to farnesol. Antimicrob Agents Ch. 2005;49(2):584-9.

15. Yuan J, Ching CB. Dynamic control of ERG9 expression for improved amorpha-4,11-diene production in Saccharomyces cerevisiae. Microb Cell Fact. 2015;14:38.

16. Grimmler C, Held C, Liebl W, Ehrenreich A. Transcriptional analysis of catabolite repression in Clostridium acetobutylicum growing on mixtures of D-glucose and D-xylose. J Biotechnol. 2010;150(3):315-23.

17. Celton M, Sanchez I, Goelzer A, Fromion V, Camarasa C, Dequin S. A comparative transcriptomic, fluxomic and metabolomic analysis of the response of Saccharomyces cerevisiae to increases in NADPH oxidation. BMC Genom. 2012;13(1):1.

18. Theobald U, Mailinger W, Reuss M, Rizzi M. In vivo analysis of glucoseinduced fast changes in yeast adenine nucleotide pool applying a rapid sampling technique. Anal Biochem. 1993;214:31-7.
19. Theobald U, Mailinger W, Beltes M, Rizzi M, Reuss M. In vivo analysis of metabolic dynamics in Saccharomyces cerevisiae: I. experimental observations. Biotechnol Bioeng. 1997;55:305-16.

20. Aledo JC, Jimenez-Riverez S, Cuesta-Munoz A, Romero JM. The role of metabolic memory in the ATP paradox and energy homeostasis. FEBS $\mathrm{J}$. 2008;275(21):5332-42.

21. Somsen OJR, Hoeben MA, Esgalhado E, Snoep J, Visser D, van der Heijden, et al. Glucose and the ATP paradox in yeast. Biochem J. 2000;352:593-9.

22. Zhang L, Nie XQ, Ravcheev DA, Rodionov DA, Sheng J, Gu Y, et al. Redox-responsive repressor Rex modulates alcohol production and oxidative stress tolerance in Clostridium acetobutylicum. J Bacteriol. 2014;196(22):3949-63.

23. Tang J, Chen $Y$, Chen $X$, Yao Y, Ying H, Xiong J, Bai J. Production of cytidine 5'-diphosphorylcholine with high utilization of ATP by whole cells of Saccharomyces cerevisiae. Bioresource Technol. 2010;101(22):8807-13.

24. Honicke D, Janssen H, Grimmler C, Ehrenreich A, Lutke-Eversloh T. Global transcriptional changes of Clostridium acetobutylicum cultures with increased butanol: acetone ratios. New Biotechnol. 2012;29(4):485-93.

25. Hüsemann MH, Papoutsakis ET. Comparison between in vivo and in vitro enzyme activities in continuous and batch fermentations of Clostridium acetobutylicum. Appl Microbiol Biot. 1989;30:585-95.

26. Wang E, Ikonen TP, Knaapila M, Svergun D, Logan DT, von Wachenfeldt C. Small-angle X-ray scattering study of a Rex family repressor: conformational response to $\mathrm{NADH}$ and $\mathrm{NAD}^{+}$binding in solution. J Mol Biol. 2011;408(4):670-83

27. Yang $X, M a K$. Purification and characterization of an NADH oxidase from extremely thermophilic anaerobic bacterium Thermotoga hypogea. Arch Microbiol. 2005;183(5):331-7.

\section{Submit your next manuscript to BioMed Central and we will help you at every step:}

- We accept pre-submission inquiries

- Our selector tool helps you to find the most relevant journal

- We provide round the clock customer support

- Convenient online submission

- Thorough peer review

- Inclusion in PubMed and all major indexing services

- Maximum visibility for your research

Submit your manuscript at www.biomedcentral com/submit 